\title{
Determination of Optimum Conditions for X-Ray Fluorescence Analysis Using Coupling Equations
}

\author{
Antonina Nikonovna Smagunova ${ }^{1}$, Oyuntsetseg Bolormaa ${ }^{2 *}$, Sergei Dimitrovich Pan’kov ${ }^{3}$ \\ ${ }^{1}$ Irkutsk State University, Irkutsk, Russia; ${ }^{2}$ National University of Mongolia, UlaanBaatar, Mongolia; ${ }^{3}$ SibVAMI, Irkutsk, Rus- \\ sia. \\ Email: *bolormuis@yahoo.com
}

Received March 29 th $^{\text {th }}$ 2012; revised April 24 ${ }^{\text {th }}, 2012$; accepted May $5^{\text {th }}, 2012$

\begin{abstract}
Coupling equations used to calculate the chemical composition of substances by X-ray fluorescence analysis can be classified as empirical, theoretical or semi-empirical based on the method for determining the coefficients of the calibration function. The advantages and disadvantages of each class of equations are discussed. Recommendations for the selecting the optimum conditions for determining empirical correction coefficients and their control during analysis are provided.
\end{abstract}

Keywords: X-Ray Fluorescence Analysis; Coupling Equation; Optimum Condition; Calibration; Reference Samples

\section{Introduction}

In the field of X-ray fluorescence analysis (XRF) calibration functions that relate the concentration of the element to be determined to the intensity of its spectral line and chemical composition of the sample are generally referred to as coupling equations. Currently, a number of coupling equations are used in practical applications. The form of these equations is often dependent on their derivation, selection of the main elemental characteristics (intensity $I_{j}$ or concentration $C_{j}$ ) and details of the determination of the correction coefficients [1,2].

Calculation of the content $C_{j}$ of element $i$ involves a complex expression for the intensity of the X-ray fluorescence of the element. Some researchers previously derived such expressions [3-6] on the basis of the fundamental laws of interaction of heterogeneous primary emission with the substance (for brevity we subsequently refer to this as "fundamental expression"), approximated by a multidimensional polynomial [7]:

$$
C_{i}=a_{0 i}+\sum_{i=1}^{n} a_{i} I_{i}+\sum_{\substack{i=1 \\ i \neq j}}^{n} \sum_{j=1}^{n} a_{i j} I_{i} I_{j}+\sum_{j=1}^{n} a_{i j} I_{j}^{2}+\cdots
$$

where $a_{0 i}, a_{i}, a_{i j}$ and $a_{i j}$ are correction coefficients, $I_{i}$ and $I_{j}$ are the intensities of the spectral lines for elements $i$ and $j$, respectively, and $n$ is the number of elements on the sample.

Depending on the method used to calculate the correc-

"Corresponding author. tion coefficients, the coupling equations can be empirical, theoretical or semi-empirical [7].

The aim of the paper is to examine the various algorithms (coupling equations) for the X-ray fluorescence analysis of materials with variable physical and chemical properties.

\section{Empirical Coupling Equations}

\subsection{Traditional Method}

Traditional methods for determining coupling equations include Equation (1) and modifications thereof. If the chemical composition of investigated samples varies only slightly, the calculation procedure can be limited to the first two terms of Equation (1). This approach is especially feasible if the effect of $j$-type elements on the $\mathrm{I}_{\mathrm{i}}$ is only due to the superposition of spectral lines. If the chemical composition of samples varies significantly, Equation (1) can be written in a non-linear form [8]:

$$
C_{i}=a_{0 i}+I_{i}\left(a_{i}+\sum_{j=1}^{n} a_{i j} I_{j}\right)
$$

Although Equation (2) is a special case of Equation (1), Lukas-Tooth and Price [8] obtained it from an expression of the fluorescence intensity excited by monochromatic radiation after representing the mass coefficient of weakening through the concentrations of $j$ elements and by replacing $C_{j}$ by $I_{j}$. In addition, the effect of sub-excitation was considered as a negative absorption. The coefficient $a_{0 i}$ takes into account the background intensity near ana- 
lytical line of $i$ element averaged over the chemical composition for calibration reference samples. This is why quantitative results using Equation (2) are obtained without background correction.

A different form of the equation was suggested by Lachance and Traill [9]:

$$
C_{i}=I_{i} / I_{i 0}\left(a_{i}+\sum_{j=1}^{n} a_{i j} C_{j}\right),
$$

where $I_{i}$ and $I_{i 0}$ are the intensities for element $i$ in the investigated and reference samples, respectively, and $C_{j}$ is the concentration of element $j$ in a sample approximated by:

$$
C_{j}=\left(I_{j x} / I_{j 0}\right) C_{j 0},
$$

where $I_{j}$ and $I_{j 0}$ are the intensities for element $j$ in the investigated sample and a reference sample with concentration $C_{j 0}$.

The values obtained for $C_{j}^{\prime}$ from Equation (4) are then substituted into Equation (3) to calculate new values of $C_{j}^{\prime \prime}$ for all of the elements. This process is repeated to derive $C_{j}^{\prime \prime \prime}$ etc, unless the following condition is satisfied:

$$
\left|C_{j}^{(m)}-C_{j}^{(m-1)}\right| \leq \Delta C_{j},
$$

where $\Delta C_{j}$ is the assumed experimental error for the concentration of element $j$ and $C_{j}^{(m)}$, and $C_{j}^{(m-1)}$ are the m and $m-1$ approximations respectively, of the concentrations.

It should be noted that if the concentration varies significantly, Equation (3) can yield divergent solutions, i.e. the value of a difference in Equation (5) does not approach $\Delta C_{j}$, but increases. This situation can be prevented by normalization for each iteration step except the last one:

$$
\sum_{j=1}^{n} C_{j}=1
$$

One advantage of Equations (1)-(3) is that they can be used in analyses of heterogeneous samples if the effect of micro absorption heterogeneity can be minimized by grinding the materials under selected optimal conditions [10]. Another advantage is the possibility of using the equations to determine chemical compositions if correction factors are only introduced for undesirable impurities [11]. In this case, the elemental composition for the main constituents is kept constant providing a so-called "containing medium". This is a prime advantage for monitoring the chemical composition of raw materials and technological products at concentrating plants, where, as a rule, the composition of non-metallic components remains constant and only the content of ore components needs to be determined.
The main disadvantage of these equations is that a large number of suitable reference samples are required.

\subsection{Determination of Optimum Conditions for Calibration of Empirical Coupling Equations}

Reference samples are required during the calibration of empirical equations. These are control samples analyzed by another method such as chemical testing. The number of reference samples $(\mathrm{N})$ required to derive the correction coefficients depends on the number of factors and we provide the following recommendations $[12,13]$.

1) The magnitude of the variation in concentration of determined and interfering components in the reference samples must be not less than that in the test samples.

2) A non-uniform distribution of reference samples in terms of the range of concentrations does not decrease the correctness of the analytical results when the analyte concentrations in the test are distributed unevenly. Thus if one equation is used to analyze several products with unevenly tests according to the range of concentrations, it is not expedient to create new reference samples by mixing the test materials for different products.

3) The correlation between the components of sample compositions of controlled object, characterized by the correlation coefficient $r_{x y}$, does not affect the systematic error of XRF results and even reduces the number of terms in the coupling equation, provided that the coefficient $r_{x y}$ does not change over time. If this condition is not met, then the variations in $r_{x y}$ require the recalibration of the equation included samples with a new $r_{x y}$ value in the number of reference samples. If the correlation between the components often changes over time, the equation is better calibrated using the reference samples for the composition of which the $r_{x y}$ value is negligible.

4) $N$ depends on the number of coefficients $(l)$ in the equations, the accuracy required (variation coefficients $\left.V_{e x t}\right)$ and errors in the chemical analysis $\left(V_{c h}\right)$ of the reference samples. These factors are linked by the following relations: if $V_{c h}$. $\ll V_{e x t}, N=l+2$; if $V_{c h} \leq V_{\text {ext }}, N \geq 2 l$; and if $V_{c h}>V_{\text {ext }}, N>5 l$.

The case $N \geq 7 l$ is not suitable, since the overall experimental error $\left(V_{c h}\right)$ for XRF does not change with the increases in $N$.

It should be noted that systematic error in the chemical analysis results (under- or overestimation of the reference sample results for a constant $\Delta C_{j}$ ) automatically affects the XRF results (i.e., they will be under- or overestimated to the value $\Delta C_{j}$ ). Thus, by increasing $N$ it is possible to decrease the influence of a random error in the chemical analysis of reference samples on the accuracy of XRF results.

Taking measured values for the intensities of analytical lines and known concentrations $C_{j}$, the coupling equation 
is constructed for each reference sample. The set of equations obtained is solved by the least-squares method to identify the coefficients in Equation (1) or Equation (2). For Equation (3) $C_{j}$, obtained to Equation (4), should be used instead of $I_{j}$.

If the concentration of target elements varies significantly in the reference samples, the modified leastsquares method should be used taking $\eta=1 / I_{i}$ or $\eta=1 / C_{i}$ as a normalization factor instead of the traditional $\eta=1$. This approach assigns a large statistical weight to samples with low concentrations of target elements, which increases the measurement accuracy in cases with low concentrations. However, it also reduces the accuracy in cases with high concentrations $[2,13,14]$. Thus, it is better to use two sets of coefficients calculated using the least-squares method $\eta=1 / C_{i}$ and $\eta=1$ to estimate low and high concentrations, respectively.

\subsection{Control of Stability for Calibrated Coupling Equations}

In view of the large number of reference samples needed for calibration of coupling equations, the analysis is not so impressive. The correction coefficients appear to be variables reflecting variations in experimental conditions. Thus, a regular control procedure is required. It should also be stressed that calculation of $a_{i j}$ coefficients using the least-squares method does not consider the real physical effects. Variations in the intensity of analytical lines within random experimental error significantly affect the coefficients. In some cases, this could lead even to a change in sign for the coefficients. Despite this effect, the concentration of a target element estimated using different coefficients might be the same (within random error). Checking of the stability of the correction coefficients is thus an essential part of experimental analysis. Checking is carried out using reference samples, with the frequency determined by the accuracy required. The lower is $V_{\text {ext }}$, the more frequent checks should be.

Registration of all the reference samples makes the calibration procedure rather complicated. To facilitate this procedure, the following steps are proposed. First, the intensity of the analytical lines is measured for all the reference samples with calculation of the correction coefficients $a_{i j}$ (i.e. calibration of the coupling equation). Then, using measured $I_{j}$, values and the calibrated equation, new conditional concentrations $C_{\text {cond }}$ are derived for the set of reference samples [15]. The use of $C_{\text {cond }}$ makes it possible to reduce the number $(k)$ of samples and thus control the stability of the equation coefficients. If the reproducibility for measurement of the intensity $I_{j}$ (equipment error $V_{e q}$ ) is significantly less than $V_{e x t}, k=l$ +2 . If this condition is not satisfied (i.e., $V_{e q}$ is only slightly less than $\left.V_{\text {ext }}\right), k=2 l$. Note that the number $k$ must include models with extreme contents of $j$ elements.

\section{Theoretical Coupling Equations}

\subsection{Method of Fundamental Parameters}

The method of fundamental parameters (FP) is based on the analyte content calculated using the fundamental expression for the fluorescence intensity. It is generally recognized that this method was proposed by Criss and Birks [16,17], but it was first introduced by Paramonov [18]. Later, the method was applied by V. Afonin and Gunicheva $[19,20]$ for the analysis of silicate in rocks and by G.Pavlinsky and Vladimirova [21] for the analysis of steel samples. The advantage of the FP method is that only one reference sample is required. As pointed out by Criss [17], only samples of pure materials (consisting of atoms of the target elements) are used for calibration. Thus, the FP method of is sometimes called "standardless", although it is difficult to agree with this, since "clean elements" are actually used as reference samples. In the FP method the intensities of analytical lines of all components $\mathrm{j}$ in the reference and control samples are measured to derive the concentration of samples using Equation (4). The concentrations obtained are then normalized by Equation (6) and serve as a first approximation of the $C_{j}$ concentrations for components in the samples. Thus, the composition determined is treated as the composition of a reference sample used for analysis of a control sample according to Equation (4). The intensity of $I_{j 0}$ is estimated using an iterative approach. First, $I_{j 0}^{m}$ is calculated according to Equation (4) and then concentration $C_{j}^{\prime \prime}$ is evaluated using Equation (5). Normalization by Equation (6) then yields a corrected value for the concentration that is used to estimate $I_{j 0}^{t}$ again according to Equation (1) and the process is repeated unless Equation (6) is satisfied. Thus, the iterative procedure matches the chemical composition of the reference sample to that of the control sample in a stepwise manner using the theoretical value $I_{j 0}^{t}$ as the denominator and the experimental value $I_{j x}$ as the numerator in Equation (4).

The FP method has never been very popular for XRF because of the complicated calculation technique. Moreover, when high accuracy is required $(<1 \%)$, the FP method, as a rule, is complemented by additional correction with the use of real samples of composition close to the test samples [22].

\subsection{Method for Theoretical Corrections}

Using the theoretical correction method high-speed computation is only necessary in the development stage of the procedure. This method was proposed by Shiraiwa and Fujino [23]. Using the intensity $I_{j}$ measured for the analytical line of element $j$ it yields the corrected value:

$$
I_{i}^{\text {corr }}=I_{i}\left(1+\sum_{j=1}^{n} a_{i j} \Delta C_{j}\right)
$$


where $\Delta C_{j}$ is the difference in $C_{j}$ between the control and reference samples and $a_{i j}$ are theoretical correction coefficients obtained as follows:

$$
a_{i j}=\left(I_{i}^{j}-I_{i}^{0}\right) /\left(\Delta C_{j}\left(I_{i}^{j}+I_{i}^{0}\right) / 2\right)
$$

where $I_{i}^{0}$ and $I_{i}^{j}$ are the intensities of the analytical lines for element $i$ calculated by fundamental expression for reference and hypothetical samples, respectively. The latter are produced on the basis of reference samples but with adjusted $C_{j}$ (the concentration of the dominant element in this sample is reduced by $\Delta C_{j}$ ). Equation (8) has a real physical meaning. The coefficients $a_{i j}$ reflect the relative change in intensity of the analytical line for element $i$ if the concentration of element $j$ in the sample changes by $1 \%$.

Hypothetical samples are produced for $n-1$ components of a sample. The intensity of the analytical lines for all $j$ elements are corrected in accordance with Equation (7) to take into account the interference effect for all elements. Then Equation (4) is applied to calculate the concentration of target elements $\left(I_{j 0}\right.$ and $C_{j 0}$ in a reference sample). If the concentration of $j$ elements varies significantly, analysis is carried out using a calibration curve obtained for theoretical intensities $I_{j}^{t}$ estimated via the fundamental expression for hypothetical samples. These samples are produced on the basis of reference samples that differ in $C_{j}$ compared to control samples within a certain margin. Concentrations are calculated using an iterative procedure.

To summarize, theoretical corrections require the development of an appropriate analytical methodology with correction coefficients and a calibration curve based on a reference sample prior to experiments. The correction coefficients obviously depend on the chemical composition of the reference sample. For example, the effect of $\mathrm{Fe}$ on the intensity of the NiKa-line is characterized by the correction coefficient, which was estimated to be $a_{\mathrm{NiFe}}=-3.70$ and $a_{\mathrm{NiFe}}=-2.56$ for regular and manganese bronze, respectively [24]. Therefore, it is desirable that the chemical composition of the reference sample is close to that of the control sample to decrease the influence of this dependence on the correctness of XRF results.

The dependence of $a_{i j}$ on the chemical composition generally leads to more complicated coupling equations. For example, Gunicheva et al. [25] proposed the following formalism:

$$
a_{i j}^{*}=a_{0}+a_{1} C_{i}+a_{i j k} C_{k}
$$

where as that of Tertian is as follows [26]:

$$
\begin{gathered}
a_{i j}^{*}=a_{0}+\sum a_{i j k} C_{k} \\
a_{i j}^{*}=a_{0}+a_{1} C_{m} /\left(1+a_{2}\left(1-C_{m}\right)\right)+\sum a_{i j k} C_{k},
\end{gathered}
$$

where $C_{m}=1-C_{i}$ and $C_{k}$ is the concentration in the third control sample of element $k$. The values of the $a_{i j k}$ coefficients characterize the effect of the third $k$ components on $a_{i j}$ and their calculation is based on ternary and binary hypothetical compositions.

\section{Semi Empirical Coupling Equations}

In this method one part of the coefficients is obtained theoretically and the other one is determined empirically. There were several such methods applied for XRF [27-30], although one is favored the method of De Jongh [30]. In this method, the coefficients $a_{i j}$ are determined theoretically by Equation (8), and the calibration curve is derived by analysis of reference samples of known elemental composition that are close to control samples.

To explain the physical meaning of the calculation procedure in the De Jongh method, consider the following case. The intensities of analytical lines $j$ elements $\left(I_{j 0}\right)$ measured for a reference sample are corrected using Equation (7) to take into account interference effects. Corrected $I_{j 0}^{\text {corr }}$ values are then used to derive the calibration curve for determining the concentration of target elements in the control sample, with appropriate correction using Equation (7).

In this method it is more convenient to use concentrations $\left(C_{j}^{*}\right)$ that subsequently corrected for the interference effect. The method is carried out as follows. For each reference sample, the normalized concentration of an $i$-type element $\left(C_{i}^{*}\right)$ is calculated as:

$$
C_{i}^{*}=C_{i}^{0} /\left(1+\sum a_{i j}^{*} C_{j}^{0}\right)
$$

where $C_{i}^{0}$ and $C_{j}^{0}$ are the concentrations of elements $i$ and $j$ in the reference sample. $a_{i j}^{*}$ is the weighted correction coefficient calculated according to:

$$
a_{i j}^{*}=a_{i j} /\left(1-\sum a_{i j} C_{j}^{0}\right)
$$

where $a_{i j}$ is the correction coefficient for element $i$.

It is worth noting that there is no unique formalism for estimation of correction coefficients. Sometimes, Equations (9) and (10), (11) can be applied, as previously reported in the literature $[25,26]$.

The next step is to determine a calibration curve derived from intensities $I_{i}$ for real reference samples and properly calculated concentrations $C_{i}^{*}$. Generally the calibration curve obeys the following law:

$$
C_{i}^{*}=a+b I_{i}+d I_{i}^{2},
$$

where $a, b$ and $d$ are constants obtained by the least squares method and $I_{i}$ is the intensity of the analytical line for element $i$ observed for a reference sample.

In experimental analysis, the measurement if intensities $I_{j}$ of analytical lines is provided for all components in a control sample and the target concentrations $C_{j}^{*}$ are 
obtained using Equation (14), which are then normalized according to Equation (6). This gives a value for the concentrations in the first iteration. Then, using a known elemental composition, correction coefficients $a_{i j}^{*}$ are calculated and concentrations $C_{j}^{\prime \prime}$ are obtained for the second iteration using the following equation:

$$
C_{i}=C_{i}^{*}\left(1+\sum a_{i j}^{*} C_{j}\right)
$$

The iteration procedure is repeated unless Equation (6) is satisfied. Concentrations $C_{i}$ are calculated using Equation (15), and the correction coefficients are obtained according to Equations (9)-(11).

Computer code Quant AS includes the algorithm [31] to determine correction coefficients for the chemical composition according to:

$$
a_{i j}^{*}=a_{1 i j}+a_{2 i j} C_{j} /\left(a_{3 i j}\left(1-C_{j}\right)\right)
$$

where $a_{1 i j}, a_{2 i j}$ and $a_{3 i j}$ are obtained using fundamental expression for ternary and binary hypothetical samples.

Further developments of the correction methodology were reported by Broll [32] and Rousseau [33,34], who used an algorithm to theoretically calculate the coefficients $a_{i j}$ from the known composition of a control sample. The method is close to the FP method, although relative concentrations are derived using an equation similar to Equation (14).

In conclusion, it is important to mention that theoretical and semi-empirical methods for coupling equations use idealized models for X-ray fluorescence from homogeneous samples. This idealization, although being continuously improved $[4,6]$, has definite limitations in that real samples are inhomogeneous. A significant increase in accuracy was observed when moving from theoretical corrections [23] to a semi-empirical approach [30], for which the calibration curve was obtained using empirical intensities $I_{i}$, or, as suggested by Molchanova et al. [35], the empirical intensities $I_{i}^{e}$ can be transformed to theoretical intensities $I_{i}^{t}$ :

$$
I_{i}^{t}=a_{0}+a_{1} I_{i}^{e}+a_{2}\left(I_{i}^{e}\right)^{2}+\sum_{\substack{j=1 \\ j \neq i}}^{n} a_{i j} I_{j}^{e}
$$

where $a_{0}, a_{1}, a_{2}, a_{i j}$ are calculated by the least-squares method for real reference samples.

Note that it is of primary importance to link the theoretical and experimental results in this step, since the theoretical approach is not as important in improving the accuracy of the correction coefficients, which are obtained from the ratio of intensities $\left(I_{i}^{j}\right.$ and $\left.I_{i}^{0}\right)$ measured for samples of similar chemical composition.

\section{Conclusion}

The analysis of coupling equations was performed fo- cusing their applicability to determination of correction coefficients. Based on this, recommendations on the optimum conditions for the X-ray fluorescence analysis for materials with variable physical and chemical properties were given.

\section{REFERENCES}

[1] Yu. G. Lavrentiev and A. I. Kuznetsova, "Coupling Equations in X-Ray Fluorescence Analysis," Zavodskaya Laboratoriya, Vol. 45, No. 4, 1979, pp. 315-326.

[2] E. I. Molchanova, A. N. Smagunova and O. F. Rozova, "Comparision of Different Types of Coupling Equations in X-Ray Analysis for Materials with Non-Permanent Composition," Journal of Analytical Chemistry, Vol. 41, No. 7, 1986, pp. 1183-1191.

[3] G. V. Pavlinsky and N. F. Losev, "Assessment of the Fluorescence Selective Excitation in Case of Mixed Primary Radiation," Journal of Technical Physics, Vol. 39, No. 9, 1969, pp. 1664-1675.

[4] A. A. Finkelshtein, "The Development of XRF Models for Homogeneous and Heterogeneous Samples," PhD Dissertation, Irkutsk, 2006, p. 40.

[5] G. V. Pavlinsky and A. Ju. Dukhanin, "Calculation of Photo-and Auger Electron Contribution to X-Ray Fluorescence Excitation of Elements with Low Atomic Number," X-Ray Spectrometry, Vol. 23, 1994, pp. 221-228. doi:10.1002/xrs.1300230507

[6] G. V. Pavlinsky, "Fundamentals of X-Ray Physics," CISP Ltd., Cambridge, 2008, 245 p.

[7] N. F. Losev and A. N. Smagunova, "The Basics of XRF Analysis," Khimiya, Moscow, 1982, 208 p.

[8] H. J. Lucas-Tooth and B. J. Price, "A Mathematical Method for the Investigation of Interelement Effects in X-Ray Fluorescence Analysis," Metallurgia, Vol. 64, No. 2, 1961, pp. 149-152.

[9] G. R. Lachance and R. J. Traill, "Practical Solution to the Matrix Problem in X-Ray Analysis," Canadian Spectroscopy, Vol. 11, No. 2, 1966, pp. 43-48.

[10] A. N. Smagunova, N. F. Losev, A. G. Revenko and A. N. Mejevich, "General Chart of Development the X-Ray Spectral Analysis Methods," Zavodskaya Laboratoriya, Vol. 40, No. 12, 1974, pp. 1461-1463.

[11] L. M. Pan'kova, S. D. Pan'kov and A. N. Smagunova, "Assessment of the Components Interference Efficiency Using the Calibration Measurements Method during the X-Ray Fluorescence Analysis," Zavodskaya Laboratoriya, Vol. 44, No. 8, 1978, pp. 950-953.

[12] A. N. Smagunova, O. F. Rozova, S. D. Pan'kov and E. I. Molchanova, "Selection of the Optimum Conditions for Setting the Calibration Function during the X-Ray Fluorescence Analysis," In: Apparatus and Methods of X-Ray Analysis, Mashinostroenie, Leningrad, Vol. 28, 1981, pp. 15-23.

[13] E. I. Molchanova, A. N. Smagunova and O. F. Rozova, "Selection of the Optimum Conditions for Setting the Calibration Function during the X-Ray Fluorescence 
Analysis," Zavodskaya Laboratoriya, Vol. 50, No. 11, 1984, pp. 25-29.

[14] R. Plesch, "Empirical Matrix Corrections in Practical X-Ray Spectroscopy," X-Ray Spectrometry, Vol. 5, No. 3, 1976, pp. 142-148. doi:10.1002/xrs.1300050308

[15] A. N. Smagunova, S. D. Pan'kov, N. F. Losev and P. I. Plotnikov, "Performing Control Over the Adjusting Factors during the X-Ray Spectrum Analysis Using the Calibration Method," Journal of Analytical Chemistry, Vol. 32, No. 1, 1977, pp. 15-20.

[16] J. Criss and L. Birks, "Calculation Method for Fluorescent X-Ray Spectrometry. Fundamental Parameters," Analytical Chemistry, Vol. 40, No. 7, 1968, pp. 1080-1086. doi:10.1021/ac60263a023

[17] J. W. Criss, "Fudamental Parameters Calculations on a Laboratory Microcomputer," Advances in X-Ray Analysis, Vol. 23, 1980, pp. 93-97. doi:10.1007/978-1-4613-3096-7 13

[18] F. P. Paramonov, "Identification of the Elements Concentration Using the Method of Theoretical Standard Selection," Commun. Acad. Sci. Kazakhstan Chem., No. 3, 1966, pp. 97-98.

[19] V. P. Afonin and T. N. Gunicheva, "X-Ray Fluorescence Analysis of Ores and Minerals," Nauka, Novosibirsk, 1977, $256 \mathrm{p}$.

[20] V. P. Afonin, "X-Ray Fluorescence Analysis of Minerals," Journal of Analytical Chemistry, Vol. 41, No. 9, 1986, pp. 1541-1556.

[21] G. V. Pavlinsky and L. I. Vladimirova, “Approximate Model for Calculation of X-Ray Fluorescence Intensity and Its Use in XRF Spectrometry," X-Ray Spectrometry, Vol. 28, No. 4, 1999, pp. 183-193. doi:10.1002/(SICI)1097-4539(199905/06)28:3<183::AID -XRS339>3.0.CO;2-9

[22] V. Ya. Borkhodoev, "X-Ray Fluorescence Analysis of Ores by the Fundamental Parameter Method," SVK NII DVO RAN, Magadan, 1999, 280 p.

[23] T. Shiraiwa and N. Fujino, "Theoretical Correction for Coexistent Elements in Fluorescent X-Ray Analysis of Alloy," Advances in X-Ray Analysis, Vol. 11, 1968, pp. 63-64. doi:10.1007/978-1-4684-8676-6 5

[24] Yu. I. Velichko, B. D. Kalinin, A. N. Mezhevich, R. I. Plotnikov and A. G. Revenko, "Dependence Analysis of Theoretical Corrections and Chemical Composition of Sample during the Steel X-Ray Spectral Analysis," Zavodskaya Laboratoriya, Vol. 43, No. 4, 1977, pp. 437442.

[25] T. Gunicheva, A. Finkelshtein and V. Afonin, "A Matrix
Effect Correction Algorithm for X-Ray Fluorescence Analysis of Steels," X-Ray Spectrometry, Vol. 19, No. 6, 1990, pp. 237-242. doi:10.1002/xrs. 1300190507

[26] R. Tertian, "The Claisse-Quintin and Lachance-Claisse Alpha Correction Algorithms and Their Modifications. A Critical Examination," X-Ray Spectrometry, Vol. 15, No. 4, 1986, pp. 251-258.

[27] G. Anderman, "Semitheoretical Approach to Interelement Correction Factors in Secondary X-Ray Emission Analysis," Analytical Chemistry, Vol. 38, No. 1, 1966, pp. 8286. doi:10.1021/ac60233a022

[28] G. V. Pavlinsky, N. F. Losev, T. N. Gunicheva and A. G. Revenko, "Method of X-Ray Fluorescence Analysis Using the Successive Corrections on the Base of Standards-Binars," In: Apparatus and Methods of X-Ray Analysis, Mashinostroenie, Leningrad, Vol. 4, 1969, pp. 184-190.

[29] A. G. Revenko, G. V. Pavlinsky and N. F. Losev, "New Variant of Calibration Method in X-Ray Fluorescence Analysis," Zavodskaya Laboratoriya, Vol. 36, No. 4, 1970, pp. 675-680.

[30] W. K. De Jongh, "X-Ray Fluorescence Analysis Applying Theoretical Matrix Correction Stainless Steel," X-Ray Spectrometry, Vol. 2, No. 4, 1973, pp. 151-158. doi:10.1002/xrs.1300020404

[31] P. A. Pella, G. Y. Tao and G. R. Lachance, "Intercomparison of Fundamental Parameter Interelemen Correction Methods," X-Ray Spectrometry, Vol. 15, No. 4, 1986, pp. 251-258. doi:10.1002/xrs.1300150407

[32] N. Broll, "Quantitative X-Ray Fluorescence Analysis. Theory and Practice of the Fundamental Coefficient Method," X-Ray Spectrometry, Vol. 15, No. 4, 1986, pp. 271-285. doi:10.1002/xrs.1300150410

[33] R. M. Rousseau, "A Comprehensive Alpha Coefficient Algorithm (a Second Version)," X-Ray Spectrometry, Vol. 15, No. 3, 1987, pp. 103-108. doi: $10.1002 /$ xrs. 1300160303

[34] R. M. Rousseau, "Corrections for Matrix Effects in X-Ray Fluorescence Analysis-A Tutorial," Spectrochimica Acta, Part B, Vol. 61, 2006, pp. 759-777. doi:10.1016/j.sab.2006.06.014

[35] E. I. Molchanova, A. N. Smagunova, T. N. Gunicheva, A. V. Smagunov, N. A. Azmuko and A. L. Pospelov, "Dependence of Correct Results of X-Ray Fluorescence Steel Analysis and Selected Method of Registering the Influence of Chemical Samples Composition on the Value of $\alpha$-Ratios in Theoretical Bind-Equation," Journal of Analytical Chemistry, Vol. 50, No. 6, 1995, pp. 595-599. 